\title{
Augmenting playspaces to enhance the game experience: A tag game case study 败弥访
}

\author{
Alejandro Moreno ${ }^{a, *}$, Robby van Delden ${ }^{a}$, Ronald Poppe ${ }^{b}$, Dennis Reidsma ${ }^{a}$, Dirk Heylen ${ }^{a}$ \\ ${ }^{a}$ Human Media Interaction, University of Twente, The Netherlands \\ ${ }^{\mathrm{b}}$ Interaction Technology Group, Utrecht University, The Netherlands
}

\section{A R T I C L E I N F O}

\section{Article history:}

Received 31 December 2015

Revised 20 March 2016

Accepted 24 March 2016

Available online 1 April 2016

\section{Keywords:}

Interactive playgrounds

Interactive tag

Ambient entertainment

Entertainment technologies

\begin{abstract}
A B S T R A C T
Introducing technology into games can improve players' game experience. However, it can also reduce the amount of physical activity and social interaction. In this article, we discuss how we enhance the game of tag with technology such that physical and social characteristics of the game are retained. We first present an analysis of the behavior of children playing traditional tag games. Based on these observations, we designed the Interactive Tag Playground (ITP), an interactive installation that uses tracking and floor projections to enhance the game of tag. We evaluate the ITP in one user study with adults and one with children. We compare players' reported experiences when playing both traditional and interactive tag. Players report significantly higher engagement and immersion when playing interactive tag. We also use tracking data collected automatically to quantitatively analyze player behavior in both tag games. Players exhibit similar patterns of physical activity and interactions in both game types. We can therefore conclude that interactive technology can be used to make traditional games more engaging, without losing social and physical character of the game.
\end{abstract}

(ㄷ) 2016 Elsevier B.V. All rights reserved.

\section{Introduction}

Play has been studied extensively for many years motivated by the positive effect it has on the development of children [1,2]. Besides being fun, play allows children to learn in a playful environment [3], develop and practice norms and beliefs [4], strengthen bonds between friends and family [5,6] or refine motor skills to understand what their bodies are capable of [7]. With the advent of digital games, PC gaming, console gaming and mobile gaming are starting to replace the traditional playground or outdoor gaming that were common a few years ago. Studies have shown that the time children spend consuming media or playing video games is often accompanied by sedentary behavior [8]. This has resulted in a major shift in children's everyday lifes, and the consequences of these changes are slowly coming to light. In most digital games, children can be seen playing "together and apart",

\footnotetext{
This paper has been recommended for acceptance by Andrea Sanna.

This paper is part of the virtual special issue on " 7 th International Conference on Intelligent Technologies for Interactive Entertainment", edited by Dr. Andrea Sanna and Dr. Matthias Rauterberg.

* Corresponding author.

E-mail addresses: a.m.morenocelleri@utwente.nl (A. Moreno),r.w.vandelden@ utwente.nl (R. van Delden),r.w.poppe@uu.nl (R. Poppe),d.reidsma@utwente.nl (D. Reidsma), d.k.j.heylen@utwente.nl (D. Heylen).
}

playing games with others but not interacting with them [9]. Moreover, digital games have also been linked to the increased amount of sedentary behavior exhibited by young children in western cultures [10]. This shift in play from traditional playgrounds towards living rooms can prevent children from developing the necessary social skills needed later in life, and can be a potential precursor for future health issues.

In an attempt to address these issues, there has been an increased focus on developing games that employ interactive technology. These games are designed to stimulate key aspects of play such as physical activity [11] or social interactions [12]. Other games aim to steer player behavior towards positive outcomes $[13,14]$. These games can take widely different forms, ranging from small interactive toys [15] to room-sized interactive playgrounds [16]. In many cases, these games are designed to make the body of the players a key component of the interaction, as this has been shown to increase a player's fun and engagement [17]. Although several guidelines on how to design such interactive games or engaging game experiences have been introduced [18-20], special consideration needs to be given to how introducing interactive technology can affect the physical and social aspects of play [12].

One way in which this can be accomplished is proposed by Tetteroo et al. in [21]. They created a design taxonomy that contains key elements of play derived from traditional children's outdoor 
game analyses and literature. With this, they propose a method to design interactive playground games. We, on the other hand, propose to augment an existing traditional playground game that already promotes social and physically active behavior through play. By enhancing the play environment in which the original game is played, we aim to improve the game experience by making it more fun and engaging without getting in the way of how the game would traditionally be played. Our approach is to analyze how the game is played, and design an interactive installation that supports the play style exhibited by the players.

In this study, we enhance the traditional playground game of tag. In tag, children adopt one of two roles: tagger or runner. When players are taggers, their goal is to chase runners and tag (touch) them. When they are runners, their goal is to avoid being tagged by the taggers. If a player is tagged, the roles of both players switch. These simple rules allow new players to understand the game quickly and join others almost immediately. However, prolonged play can lead to boredom since there are no win conditions. By introducing technology into the playground, we envision a plethora of opportunities to enhance the game which would otherwise not be feasible.

In this paper, we first describe a pilot study where children were asked to play tag in an uninstrumented open space. The game sessions consisted of different versions of tag games, played with a varying number of players. These game sessions were recorded and analyzed to derive characteristics of how the game is played [22]. Based on these findings, we designed and implemented the Interactive Tag Playground (ITP), an interactive game installation that aims to enhance the game experience of traditional tag games while allowing players to exhibit playful, physically active and social behavior [14,23]. The ITP tracks players inside the playground, display game elements on the floor, and guide interactions by processing the game logic. By doing this, the ITP becomes de facto a referee, enforcing rules for the players in situations where disagreements arise. Additionally, the ITP facilitates the analysis of player behavior by logging automatically collected player data such as position and role. By using this information in-game, researchers can explore different gameplay interactions and elements and quickly see how they affect player behavior. By using it offline, researchers can carry out player behavior analyses without the need of annotation, significantly reducing the time involved in the process.

To evaluate our playground, we conducted two user studies in the ITP where we asked participants to play both traditional tag and interactive tag. The first study was conducted with adults, the second study with children. In both studies, we evaluated three aspects of the ITP. First, we evaluated whether the ITP enhances the game experience of traditional tag games. Second, we checked whether the augmented tag game allows players to demonstrate physically active and social behavior. Third, we analyzed specific behavioral cues important in tag games to showcase the potential of the ITP as a game research platform. We accomplished this using questionnaires, semi-structured interviews, observations, and objective analysis of the data automatically collected by the ITP.

This article is structured as follows. In the following section, we present a short overview of how technology is used to enhance games, and point out design considerations. In Section 3, we describe our study of traditional tag games, highlighting the requirements needed to build an installation that supports this style of play. In Section 4, we introduce our interactive installation, the Interactive Tag Playground. In Section 5, we describe our two user studies, the results of our observations and the discussions with the players. We also present the results of the game experience evaluation and the player behavior analysis of the interactive tag games. Finally, we summarize our findings and discuss avenues for future work in Section 6.

\section{Enhancing games using technology}

Besides creating novel games, technology can also be used to enhance the user experience of traditional games. For instance, the "BuzzTag" installation provides players the opportunity to play games similar to traditional tag games, but using interactive devices to enhance the interaction [24]. One of the main goals is to maintain face-to-face social interactions during play. To this end, they use wireless devices that provide haptic, visual and auditory feedback. In "BuzzTag", the player who possesses the "buzz" needs to get rid of it by tagging other players. Another example is the "Table Tennis for Three" game installation designed by Mueller et al. [25]. The goal of their installation is to allow players that are not physically colocated to play table tennis together. Furthermore, instead of just playing against one other player, you play against two.

Technology can also be used to enhance games by modifying gameplay elements based on automatically measured player data. For example, heart rate measurements have been used to change game elements in the "Beats Down" mobile game [26]. Their game concept resembles the well known "whack-a-mole" game: given a set of tiles, the user needs to quickly tap the tiles that are flashing. There are two variations of the game. In challenge mode, heart rate is used to modulate the flashing frequency, allowing players to get more points by increasing their heart rate and, therefore, the number of flashing tiles. In relax mode, players get point multipliers that increase as the player's heart rate decreases. Navarro et al. also use heart rate measurements to adapt actions in-game in the "Webz of Wars" game [27]. Right before the game starts, a baseline heart rate measurement is taken. Afterwards, in-game heart rate measurements are used to scale the power of a player's attacks. The higher the heart rate is in comparison to the baseline, the more effort the player is putting in, the more powerful the attack gets. Landry and Pares also look at player exertion, but propose computer vision algorithms to measure it in the "Interactive Slide" [28]. The installation consists of an inflatable slide equipped with a camera projection system, in which the cameras are used to track players and the projectors are used to display the game elements onto the slide. By measuring the amount of movement of groups of players, the system is able to adjust the tempo of the game, triggering different amounts of physical activity.

One specific type of gameplay adaptation that has been used in many studies is skill balancing. Skill balancing aims to help people with different skills play together by changing game elements. The adaptation can be achieved by automating processes based on in-game measurements, or offline based on previous player information. The latter, for instance, was used by Altimira et al. when attempting to balance player' skills in Wii table tennis [29]. Players were asked beforehand to assess their own skill at table tennis, and the best players were given handicaps before the game sessions. To balance skills using automated measurements, data can be obtained from external sensors or from the game itself. Stach et al. balance the difficulty of a cycling exergame using heart rate monitors [30]. The "Heart Burn" racing game allows people of different fitness levels to compete not by measuring the speed at which they cycle, but the effort they put in as measured by their heart rate. This information is used to scale their in-game performance. On the other hand, Vicencio-Moreira et al. use in-game data to balance the skills of opposing players in a first-person shooter [31]. They use two methods to achieve this: "Bullet Magnetism", which makes bullets home in on the target within a given activation range, and "Area Cursor", which increases the activation area for a target hit. The assistance is given to the player with the least number of kills, in proportion to how far behind from the other players he is. 
One important aspect that needs to be considered when introducing technology into games is the effect it can have in the inherent benefits of traditional play. Isbister argues that in real life we connect through physical experiences, and that games should not be any different [12]. She mentions that the use of screens or projections could limit the amount of social interaction between players. Sturm et al. agree with this, saying that embodied games can and should stimulate group play and social interactions, both of which are strong motivators for engaging in physical activity [32]. For example, Lindley et al. show that embodied games can help increase engagement as well as enhance social interactions when compared to playing with traditional controllers [33]. Sometimes, though, designing interactive systems that support the positive aspects of play is not enough. In [17], Berthouze studies how body movement and engagement in games are related, and discusses the difference in strategies with which players approach a game: "hard-fun" and "easy-fun". In the hard-fun strategy, players are solely concerned with winning and will exploit technology to their advantage. For instance, during the analysis of several embodied games, they saw hard-fun players limiting their movements to the bare minimum since winning was easier when moving less [34]. This shows that planning carefully how to introduce technology in games is necessary, but so is studying how players interact with the technology once it is in place.

Instead of developing a game that is designed specifically to promote social interactions or physical activity, we propose using technology to augment an existing playground game that inherently promotes these key aspects of play. We start by analyzing the way the game of tag is played traditionally, and design an installation that supports the style of play exhibited by the players. In doing so, we enhance the game experience without limiting players' ability to express themselves socially or physically. This is accomplised by allowing players to interact with each other, and with the system, in a natural way without the assistance of wearables or tangible play objects as used in other studies [24,35]. Additionally, our installation facilitates the collection of player data during the game to enable the design of novel game interactions as well as the analysis of player behavior. We achieve this by using cameras, which have the added benefit of allowing players to move freely during play. This approach is similar to what has been attempted in $[21,28]$. We, however, do not require tags to detect players and are capable of tracking players individually as they move in the playground.

\section{Analysis of traditional tag games}

We decided to augment the game of tag as children play it regularly. To understand how to enhance the game, we conducted a pilot study where we asked children to play different versions of traditional tag in an uninstrumented space. These game sessions were recorded, annotated and analyzed to get insights on how the game is played, its shortcomings, and possible ways to enhance it.

\subsection{Play corpus}

We recorded the Play corpus, a dataset that contains children playing different versions of tag games. ${ }^{1}$ The game sessions were played in an open space of $7 \times 6 \mathrm{~m}$. The children that took part in these recordings were between 8 and 12 years old. Besides normal tag, the children also played three variations of the standard game. In this study, we focus our analysis on the standard version of tag.

There were 9 sessions of normal tag, which lasted in total 12 and a half minutes. During this time, 74 tag occurrences took place,

\footnotetext{
1 The Play corpus can be obtained from http://hmi.ewi.utwente.nl/playcorpus.
}

which means that, on average, there was one tag every $10.14 \mathrm{~s}$. A maximum of 8 players could play simultaneously in each session. One researcher acted as a referee during the tag sessions. The role of the referee was to instruct players to enter or leave the playing area, assign the role of the tagger at the beginning of each game session, and start or stop game sessions. As such, the duration of the game sessions and the number of players taking part in each session vary.

To record the game sessions, we used two methods. For the first method we used three RGB cameras, which were located outside the playing area forming an equilateral triangle. This meant that all children were visible from at least one camera during the entire game session. For the second method we placed four Microsoft Kinect sensors on the ceiling of the playing area to capture top-down depth images of the game sessions. These images were manually stitched together and used as the basis for our offline, semi-supervised tracker. A moving median filter was applied on the position data to smooth out the noise on the tracked positions. The roles of the players were manually annotated by only one person given the objective nature of the data.

\subsection{Insights of traditional tag games analysis}

During the recording and analysis of the Play corpus, several important observations were made. These observations helped us identify aspects of the game that we should try to maintain, and potential challenges we would need to deal with when enhancing the game of tag. These are detailed below. We also briefly describe the process by which the behavior was analyzed, to showcase how it could be improved in an interactive installation.

\subsubsection{Player behavior}

The players were highly active during all game sessions. Many players really exerted themselves when running to tag other players, or to run away from them. Besides running, players also slid on the floor, jumped, ducked or pushed other players around in an attempt to prevent being tagged. We never saw children walking or losing interest in the game due to exhaustion, but this could be because we allowed them to rest by bringing other children into the game while they waited just outside of the playing area.

We also saw players interact with each other often and in many different ways. The most common one was verbal communication, for instance, to ask who the tagger was or jest about the game. Nonetheless, players also taunted each other, or tried to get revenge when tagged. Some players also exhibited acts of deception, as they pretended not to be taggers to make it easy to tag other players, or pretended not to be tagged because they wanted to be runners.

Players had a lot of fun during the game. They looked happy and engaged throughout most of the sessions, with a couple of exceptions due to some players not being very good at tag. A lot of yells and laughter could be heard during the sessions. Also, some actions unrelated to the game could be witnessed, such as players dancing or pretending to swim on the floor.

\subsubsection{Tag game shortcomings}

There are several events that can disrupt the flow of the game, or outright cause the game to end before players meant to stop playing. We call these break-downs of play. We observed several of these during the recording of the Play corpus, the most common one being losing track of who the tagger was. When the game started, it was fairly easy for everyone to identify who the tagger was. However, as the game progressed, children started running with their backs to other players, which resulted in them being unable to identify who the tagger was afterwards. This was especially evident in sessions where the number of players was high 
(6-8 players). Additionally, we observed players pretending not to be the tagger, walking close to someone, and then tagging them. Sometimes, players cheated saying they had not been tagged. When confusion ensued, the game sometimes would break down.

Another problem that sometimes led to play breaking down was the difference in abilities between some players. Certain players were quite slow in comparison to other players, and thus had difficulty tagging. After several unsuccessful attempts, they would slow down and eventually give up. This is understandable as frustration builds up from not being able to tag others. This not only affects the tagger, but also the runners that are not being challenged. They would also start to slow down and eventually taunt the tagger to attempt to restore the interest in the game. This sometimes helped temporarily, but after some unsuccessful attempts, apathy kicked in again. In many cases, the referee had to assign a new tagger or recruit an additional tagger from the pool of players and restart the session.

\subsubsection{Difficulties processing the data}

To analyze the behavior of the players, we had to manually process the data, even though we had automated certain processes. For instance, we implemented a semi-supervised tracker to help us with the acquisition of the position data for the players. The depth information from the Kinects allowed us to separate background (floor) from foreground (players) and detect and track each player in the playing area. Usually, tracking results were propagated automatically. However, when players got too close to each other, their contours would merge, resulting in the tracker detecting them as only one player. Since each player was given a unique label at the start of the recordings, when this happened, it was impossible for the tracker to consistently assign the correct label to the merged players once they split up. In these cases, manual input was requested by the system. Missing detections were interpolated if they were missing for less than three seconds, otherwise manual input of the location was needed as well.

RGB cameras were used to manually annotate the roles of the players. An annotator had to go over the video several times as many players did not behave according to their role. This was witnessed often during tags. Some players would pretend they had not been tagged, which meant the previous tagger was confused as to whether he had to continue being the tagger or not. Sometimes, the tag was not noticed by the tagged player, and the same situation ensued. In both cases, usually the player who had been the tagger would revert to the tagging role after a short period of time. Sometimes it was not clear if someone had been tagged due to occlusion, even when having three different cameras.

\subsection{Requirements for enhancing tag game spaces}

One of the reasons to collect the Play corpus was to understand the behavior analysis process and how the game is played. Below, we present several design requirements for our interactive tag game installation derived from the insights gained from analyzing player behavior in the Play corpus.

\subsubsection{Fun and engagement}

The main requirement of an entertainment installation is to provide an engaging and fun experience. One way to achieve this is by enhancing the original game of tag by adding game elements that introduce variety to the game. Since we do not want to affect player behavior, we need to employ methods that allow players to behave as they normally would. Following this, the use of projections is a good approach, as additional information can be displayed onto the playing area without disrupting play. Ambient speakers would also work towards this goal.
Another way to maintain player engagement is by adapting game mechanics as studies have shown that games that always play out the same can become boring [36]. As such, the playground should be capable of adapting its gameplay based on cues sensed from each specific play-through. This would guarantee different experiences since gameplay would change in an ad-hoc manner. A specific example of this is skill-balancing, which would be very useful in tag games as big disparities in skills led to the breakdown of play in the Play corpus. Although learning to deal with these issues is an important aspect of play, when left unchecked, players can become annoyed or irritated.

In this regard, the playground should aim to minimize the chances of play breaking down, as they can detract from the overall game experience. Besides skill disparities, other common causes for the break-down of play were players being confused on who the tagger is, or people pretending to not have been tagged. The system should, at the very least, try to prevent these issues. This could be achieved by mediating the interactions between players and the game.

\subsubsection{Unobtrusive and autonomous functioning}

An important design consideration for the development of our installation is how to measure player behavior without obstructing play. If players cannot play as they want, this would inevitably lead to diminished levels of engagement and less natural behavior. Since the playground should be able to run autonomously in public spaces, we should not rely on wearable devices to gather player data as researchers would need to be present to hand them out and retrieve them. Using cameras to sense player behavior seems a valid solution, as they can be used to measure behavior while giving players the opportunity to play without restrictions. The cameras should be placed such that they are not in the way of the players.

Since the game of tag supports the concept of players joining and leaving the game as they wish, our playground should also support this easy-in, easy-out style of play. The playground should be able to locate and track players that walk into the playing area and add them to the game immediately. On the other hand, as soon as they walk out, the system should remove them from play without causing any disruption for the remaining players.

\subsubsection{Physically active, social behavior}

Tag games are especially effective at encouraging players to exert themselves through running and chasing as key game mechanics. To be able to retain this characteristic, players should be allowed to run freely inside our interactive playground. Also, since tagging is achieved by getting physically close to other players and touching them, our playground should be capable of detecting the position of each player in any given moment of the game.

Players also exhibit a wide array of social behavior during tag games, such as talking, joking, taunting, etc. As such, our installation should also support these types of social behavior. This means players should be capable of communicating verbally and physically while playing.

\subsubsection{Automation of tasks and data measurements}

During the analysis of the Play corpus, player data had to be manually annotated using video recordings. This process is timeconsuming and error-prone. If some, or all, of this information could be collected automatically by the game installation, the behavior analysis process would be significantly shortened. Not only could this information be used post-game, but, if gathered in real-time, it could be used in-game to react to specific events. Therefore, our playground should directly obtain this information. 


\section{The Interactive Tag Playground}

Based on these requirements, we developed the Interactive Tag Playground, an interactive game installation that uses sensor, sound and projection technologies to enhance traditional tag games. The ITP uses cameras to track players, projectors to display visualizations on the floor of the playground, and PCs to process the game logic and guide player interactions during the game (Fig. 1). By automatically logging player data such as position and role, the ITP doubles as a tool that facilitates research on how game elements can affect player behavior and play experiences.

The ITP consists of four Kinects located in the ceiling of the playground. The Kinects are situated in a grid-like setup, $4.0 \mathrm{~m}$ apart from each other. Two projectors are also located in the ceiling, $4.0 \mathrm{~m}$ apart, in between the Kinects (Fig. 2). The ceiling is situated at $5.3 \mathrm{~m}$ above the playing area, which allows us to track players in a $7 \times 6 \mathrm{~m}$ area. With the two projectors, we cover an area of approximately the same size. Speakers are used to produce sound effects during the game, and can be located either next to the playground or suspended from the ceiling. With this setup, the ITP is capable of supporting an easy-in, easy-out style of play, which fits its potential use as a public installation.

At the start of each game, a tagger is chosen randomly from the detected players. If the tagger leaves the playing area, the system randomly chooses one of the remaining players as the new tagger. To indicate the role of each player, the ITP displays colored circles underneath the players: orange for taggers and blue for runners. We use neon colors with an overly bright effect to achieve a look that is appealing to children and well suited for projection onto dark floors. These circles also leave bright trails behind them upon movement (Fig. 3). We do this to make it clearer for the players that their movement is being tracked and that it has an impact on the game. The circles pulsate slightly to make it look as if they were alive, making the game more dynamic.

When the game begins, the circles enter the playing field and move towards the players to let them know that each one of them has a circle that will follow them during the game. Likewise, whenever a new person enters the field, a new circle will appear and move towards the new player. These circles play an important role in the game since, instead of physically tagging other players, the tagger has to get his circle to overlap with a runner's circle. When this happens, the color of both circles switch to indicate that the roles have changed. A sound is also played to indicate this event. If a player is tagged, he is not allowed to tag the previous tagger back for two seconds, enforcing a cool-down period. To make this clear to the players, their circle becomes slightly transparent. This encourages players to look for other players to tag. As such, the installation is de facto a referee, capable of enforcing rules to prevent disagreements between players. Fig. 4 shows an overview of the ITP architecture. The most important components and tasks are described subsequently.

\subsection{Player tracking}

Our online, top-down, multi-person tracker uses the depth images from the Kinects as input to detect players. We only use depth images because the game projections are better appreciated in dark environments, which would make the use of RGB images difficult due to the uneven and low illumination conditions. An overview of the tracking process can be seen in Fig. 5. Player identity cannot be estimated from the low resolution, top-down depth images. By default, depth images are not stored by the system to protect the privacy of the players.

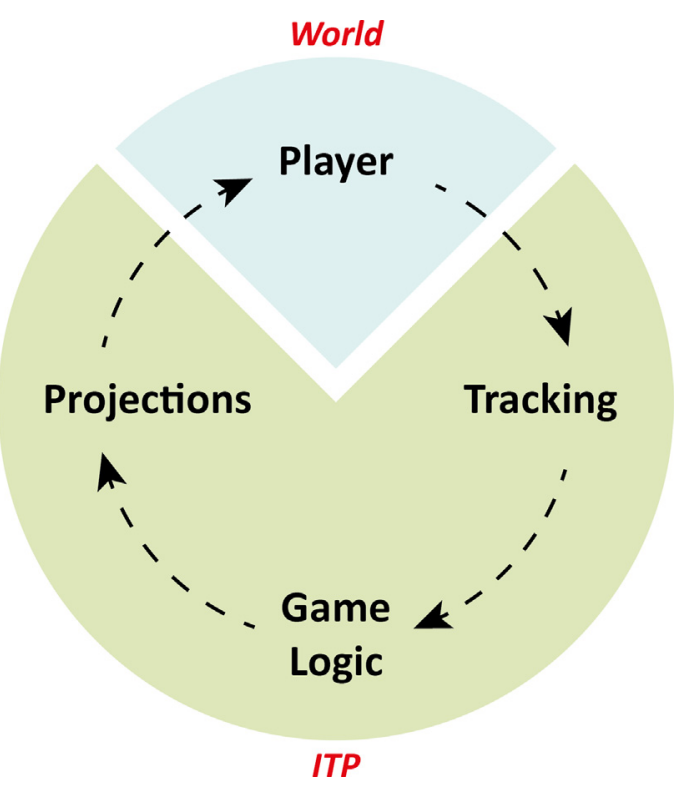

Fig. 1. Interaction between the ITP elements.

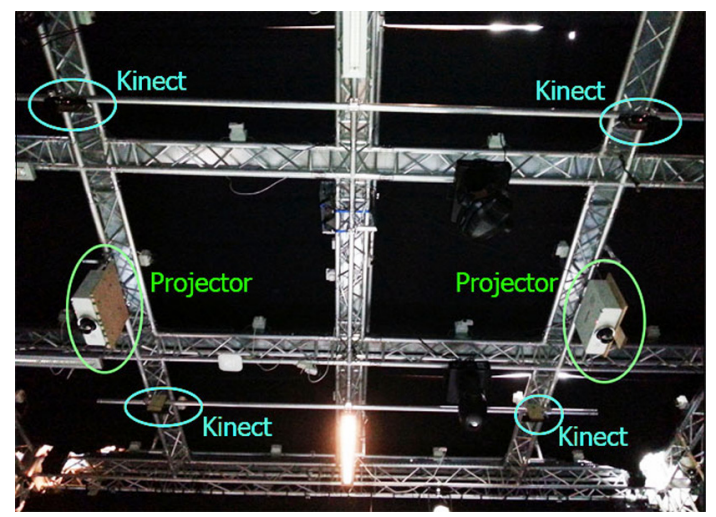

Fig. 2. Physical arrangement of the different hardware elements on the ceiling of the ITP.

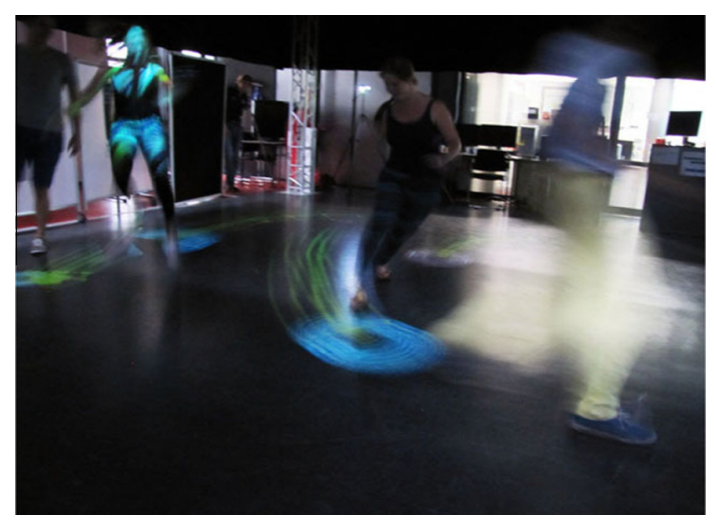

Fig. 3. Young adults playing tag in the ITP.

We detect the location of the players by applying a threshold to the depth images to remove the floor and small objects that might be present. The value of the threshold is chosen based on the height of the players we want to track. We promote the head and shoulder regions of the players by filtering the thresholded images with a Difference of Gaussians kernel. Afterwards, we find the contour 

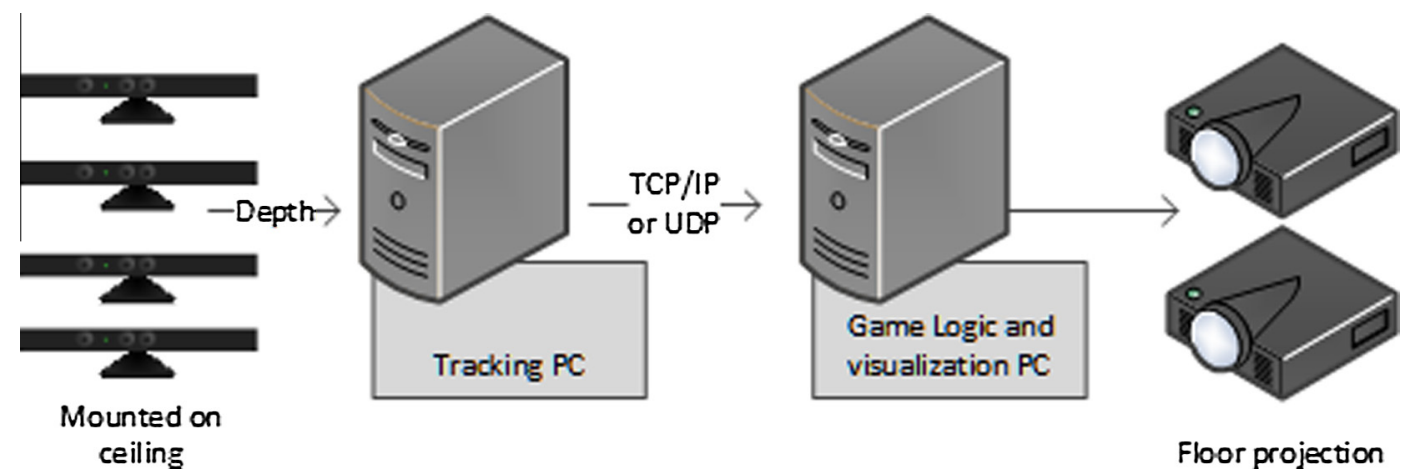

Floor projection

Fig. 4. Overview of the ITP's architecture.

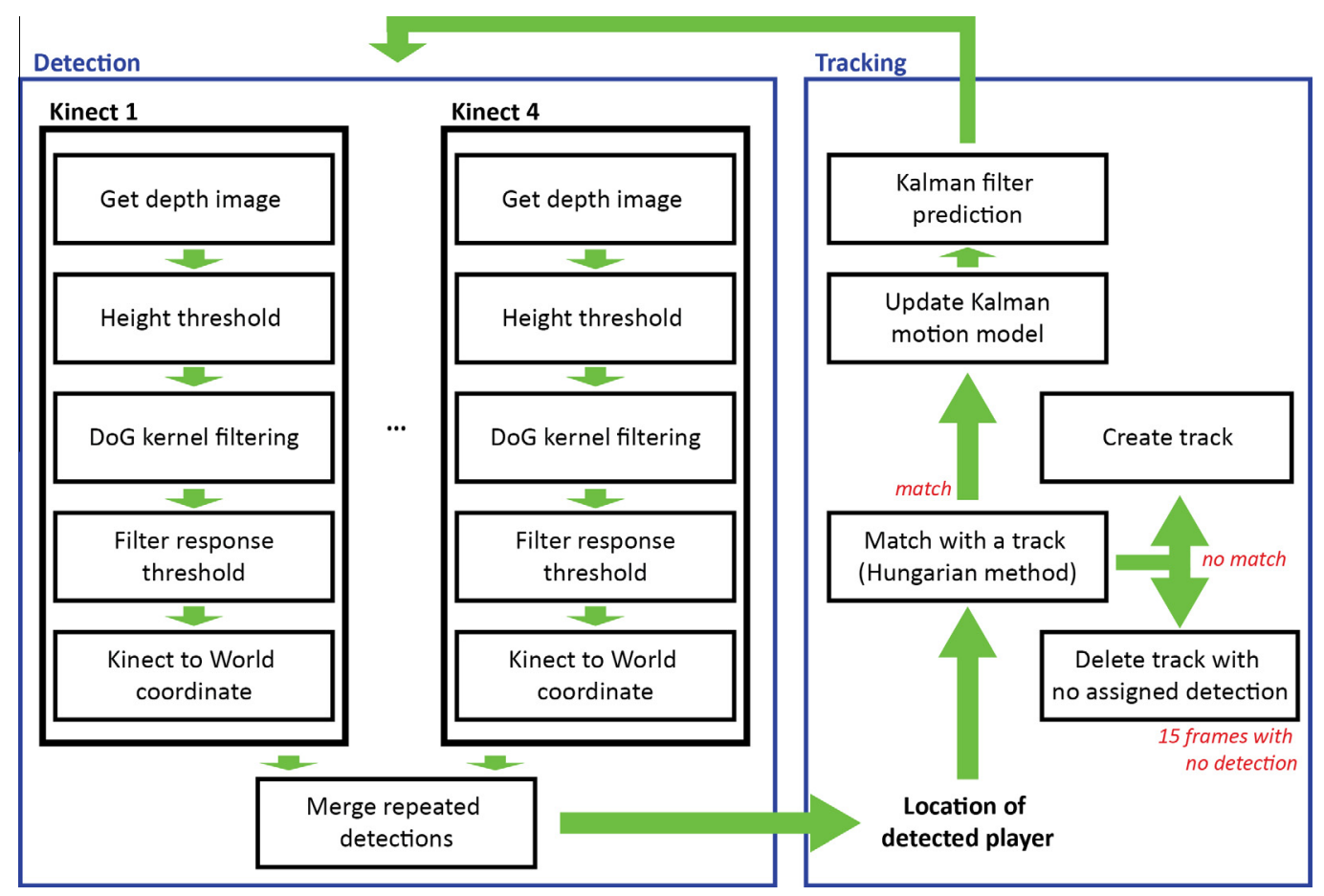

Fig. 5. Overview of the tracking algorithm.

of the areas with a high response to our filter, which typically correspond to players, and find their centers of mass. Once we have the centers of mass, we map the Kinect-specific coordinates (pixels) to real-world coordinates (meters). This is done for each Kinect. Finally, we merge detections that originate from different Kinects but are within $0.5 \mathrm{~m}$ of each other to correct for overlap in the Kinects' field of views. This overlap is needed to make the tracking more fluid when players move between Kinects.

Once the players have been detected, we use Kalman filters [37] to track them in the playing area. The algorithm behind this filter works recursively in two steps: a prediction and an update step. During the prediction step, a Kalman filter tries to predict state variables (e.g. position) based on their history (e.g. past positions) and the estimated system model (e.g. laws of motion). During the update step, newly measured values are used to correct the prediction and update the system model. Detections are matched to the tracks using the Kuhn-Munkres algorithm. This method minimizes the cost of assigning detections to tracks which, in our case, consists of the Euclidean distance between them.

When a new detection is found, we wait for five frames (approximately $0.25 \mathrm{~s}$ ) to make sure it is an actual player and not camera noise before creating a track for it. When a track has no assigned detection in a given frame, we wait 15 frames (approximately $0.75 \mathrm{~s}$ ) before deleting it since most of the times it is a player that went outside the field or below the threshold temporarily. By doing this, we prevent creating unnecessary tracks or deleting valid ones.

\subsubsection{Tracker performance}

To evaluate the tracker, we recorded and manually annotated thirteen tag game sessions of one minute and a half. Four children played simultaneously in each session. We use two metrics to evaluate the tracker. The first one evaluates the chance that a player's track would switch with that of another player. The second one evaluates the chance that a player is lost by the tracker inside the playing area. The results can be seen in Table 1 .

Overall, the performance of the tracker is good. Out of 10 tag occurrences, only one results in a track change between players. On average, there are only two track switches per session i.e. one every $45 \mathrm{~s}$ of play. Also, the low number of track losses inside the playing area indicate that the tracker is very reliable in locating players. 
Table 1

Evaluation of the ITP's tracker.

\begin{tabular}{ll}
\hline Number of frames & 21877 \\
Number of tags & 265 \\
Probability of track switch & $9.81 \%$ \\
Percentage of time a player is not tracked & $2.63 \%$ \\
\hline
\end{tabular}

\subsection{Distributed components}

To simplify the process of designing and implementing interactive rules or elements, the game logic is separated from the tracking system in the ITP. This is achieved by having the game engine and the tracker in separate computers that communicate over a network. The tracker PC sends the position of all players to the game $\mathrm{PC}$, which uses this information to drive the game interactions. Since we can distribute tasks, it would be possible to use additional computers to manage other tasks (e.g. projections, sounds) or extend the effective playing area.

\subsection{Data logging}

The ITP can log the position and role information of all players in real-time. This information can be used in-game to drive certain game mechanics (e.g. display a circle underneath a player's location), or after the game to analyze player behavior (e.g. analyze how players move during the game) or evaluate whether goals are met (e.g. measure the amount of physical activity).

\subsection{Interactive tag games}

By manipulating different gameplay elements of the interactive tag game, player behavior can be steered in different directions. We have tested three different approaches: tag with dynamic circle size, tag with arrows pointing to certain runners, and tag with power-ups. Each intervention is aimed at promoting specific player behavior [14]. Considering that we have also implemented a version of interactive tag that has no interventions, four different types of tag can be played in the ITP. In this article, we use the tag game with the dynamic circle size intervention, and the tag game with no interventions. The dynamic circle size intervention aims to balance players' skills by changing the size of their circles depending on the time a player has been a tagger (Fig. 6). When a player is a tagger, his circle will slowly grow (up to a maximum size) as long as he remains the tagger, making it easier to tag other players as time goes by. On the other hand, when a player that has been a tagger for extended periods of time becomes a runner, his circle shrinks (down to a minimum size), making it easier to avoid getting tagged.

\section{Evaluating the Interactive Tag Playground}

The ITP has three distinct goals. First, to enhance the game experience of traditional tag games by using interactive technology. Second, to support natural tag game behavior: it should allow players to exhibit physically active and social behavior. Third, to facilitate player behavior analysis. To evaluate these goals, we conducted two user studies, one with adults, and one with children. In both cases, players played traditional (uninstrumented) and interactive tag and were asked to share and compare their experiences. We also analyzed their behavior using the data that the ITP automatically collects.

Our user studies were carried out in different locations, and therefore we had to adapt the setup to fit the available space. The two setups are almost identical, with only small variations. The setup used in the second user study is slightly smaller, with a $6 \times 5 \mathrm{~m}$ playing field. Due to the smaller size, the tagging cooldown was reduced to $1.5 \mathrm{~s}$. The circles were also static instead of pulsating. Lastly, a bright green border was drawn on the boundaries of the playing field to prevent players from going out of bounds.

\subsection{First user study: adults}

For our first user study, we recorded seven play sessions, each one consisting of both traditional and interactive tag subsessions. The participants were recruited from around the university and were all PhD, master or bachelor students, except for two participants which were lab managers. Except for one session in which five players played simultaneously, the sessions consisted of four players playing tag. In total, 29 users took part in the study. Each sub-session lasted three minutes, for a total of six minutes of tag per group. All sessions were played in the ITP, but the traditional version of the game was played with no sounds or visuals. The interactive tag game version that was played was the dynamic circle size adaptation version.

After filling in a consent form, the players were explained that they would play two different games of tag: traditional tag and interactive tag. The order of the sub-sessions was alternated between groups. When explaining the game, players were not informed of the dynamic circle size intervention. There was a short break of approximately one minute between sub-sessions. Once the session ended, players were asked to fill in a questionnaire to compare both tag games. The questionnaire also asked how players felt about the interactive elements of the ITP, and whether they thought the system was fair even with the adaptive circle size intervention. In one session, only two participants filled in the questionnaire, which resulted in only 27 participants evaluating their experience. After filling in the questionnaire, players were invited to a short discussion and feedback session.

Below we present our questionnaire, explain how it was constructed, and discuss the players' ratings. Then, we describe our observations of the game sessions as well as the comments we received from the players in the feedback session.

\subsubsection{Questionnaire}

We designed a questionnaire (see Table 2) based on the Revised Gaming Engagement Questionnaire (GEQR) of Berthouze [17]. Our questionnaire consists of four dimensions. The first two compare the game experience of interactive tag and normal tag (A-Enjoyment, B-Immersion). The last two dimensions evaluate elements of the ITP (C-Gameplay, D-Enjoyment of game elements). The questionnaire has two additional categories (Balance/Fairness, Skill level) that do not necessarily measure the same construct, but evaluate related issues interesting for our study.

Out of the 24 questions in the GEQR, we chose 16 that were relevant to our research interests and fit our game (questions 3-18) since it lacked narrative. We also added 11 questions of our own (questions 1-2 and 19-27). We used a Likert scale which ranged from 1 (Disagree) to 7 (Agree), and therefore had to rephrase the GEQR questions from How much...? to statements for which the participants had to specify their level of agreement. Furthermore, the GEQR questions used for the first two dimensions had to be rephrased to accommodate the comparison of the tag conditions.

In the printed questionnaire, all questions were put in a constant randomized order. Our modified version of the questionnaire has not been validated, but we do present the Cronbach's alpha for each dimension. Q14 and Q22 are reversed ( $r$ ) when calculating the dimension statistics because of their direction with respect to the other questions. Table 2 shows the reversed scores. 


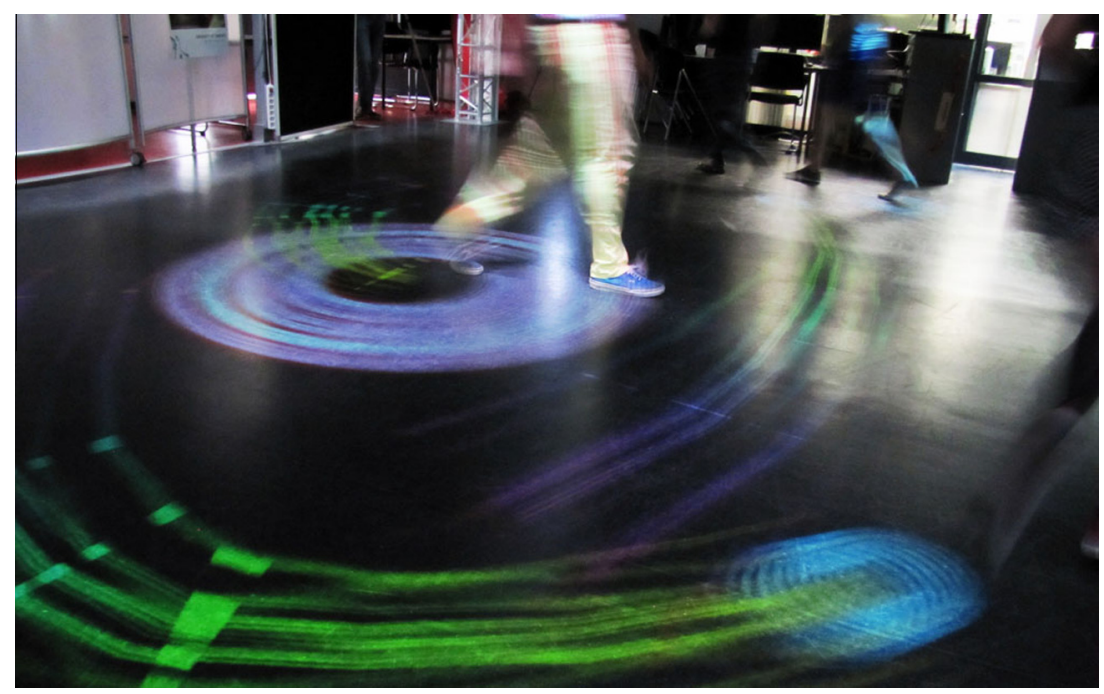

Fig. 6. An instance of the tagger's circle having grown in size.

5.1.1.1. Enjoyment (A). The Enjoyment dimension evaluates whether players had more fun playing interactive tag than normal tag. It consists of five questions (Q1-5). Given the scale we use, a value close to four implies players do not enjoy one tag game over the other. We calculate the Cronbach's alpha to check for consistency in the answers, and obtain a value of 0.88 . This means that the questions indeed measure a single construct. The mean of the answers for the enjoyment dimension is 5.37, and the standard deviation $(\sigma)$ is 1.06 . This indicates an effect towards interactive tag. To find out if this effect statistically significant, we conduct a two-tailed one-sample $t$-test against the center of the scale. This shows a significant effect $(t(26)=6.7, p<0.001)$ in the direction of more enjoyment during the interactive sessions. Therefore, we can state that the players enjoyed the interactive game more than playing traditional tag.

5.1.1.2. Immersion (B). The Immersion dimension evaluates whether players lose themselves while playing interactive tag more than they do when playing normal tag. It contains four questions (Q6-9). Like the previous dimension, a value close to four implies that players do not get more immersed in one condition over the other. Cronbach's alpha is 0.71 , implying that there is a fair correlation between the items. The mean for the answers is 4.93 , with $\sigma=0.93$. We use a two-tailed one-sample $t$-test against the center of the scale to check for statistical significance, and the result shows a significant effect $(t(26)=5.2, p<0.001)$ in the direction of more immersion during the interactive session. Thus, players were more immersed during the game of interactive tag compared to traditional tag.

5.1.1.3. Gameplay (C). The Gameplay dimension evaluates whether the different game elements and mechanics are understood and how the game is played. It consists of six questions (Q10-15). The Cronbach's alpha for this dimension is quite low at 0.48 . Due to some network issues during the user study, players experienced some lag in the circle movement. We believe the low alpha is a consequence of this, as gameplay is positively affected when delays between actions and the system's response are small. Indeed, if we remove the questions affected by the small delay (Q10 and Q14), the value of alpha increases to 0.74 .

The mean for this dimension is $4.96(\sigma=0.56)$. If we remove the two questions mentioned above, the mean is $5.82(\sigma=0.71)$. We can see that the lag has a strong effect on the Gameplay scores.
However, even with the questions affected by the lag, the mean is still leaning towards the positive scale of Gameplay. Players felt good about how the game played out. However, the lag problem needs to be solved.

5.1.1.4. Enjoyment of game elements (D). The Enjoyment of game elements dimension evaluates if players liked the graphics, sound and theme of the game. It is composed of three questions (Q16-18). The Cronbach's alpha for this dimension is 0.35 . The low alpha value is due to Q17, which asks about the audio effects of the game. Regrettably, many players did not hear any sound being played during their playing time. This may be due to the volume being too low, or the speakers not being placed properly.

The mean of this dimension is $5.31(\sigma=0.75)$. Without Q17, the mean increases to $5.61(\sigma=0.79)$ and the alpha to 0.63 . This alpha value is still not very high, which may indicate that these questions would fit better in different dimensions. Regardless, under the current structure, the results convey that players enjoyed the game elements overall.

5.1.1.5. Balance/Fairness. The category of Balance and Fairness evaluates if the players felt the ITP allowed them to play tag as they would have liked, and whether they thought it was helping players based on their skills. It consists of four questions (Q19-22). Q19 and Q22 ask whether the ITP allows players to play normally and have a mean of 4.11 and $4.41(\mathrm{r})$ respectively. This conveys two things. First, players felt the game did not interfere with how they wanted to play. Second, it did not allow them to showcase all their abilities. It would have been ideal if the players felt the system allowed them to demonstrate all their tagging skills, but it is still important that they do not think the game limits their ability to play the game.

Q20 and Q21 deal with the issue of Balance, and whether players feel anyone was receiving help from the ITP. A high value indicates that players felt the game helped a particular type of player. The mean for Q20 is 4.63, which shows that players had a feeling that the ITP was helping less skilled players, but in overall were not very pronounced in their judgment. One reason for this may be that, due to the short game sessions, the circle growth rate was accelerated and therefore was more noticeable. In regards to skilled players, the mean for Q21 is 4.04, which implies players did not feel it hurt or helped skilled players. We believe these 
Table 2

Questionnaire used in the evaluation of the ITP, with means per question and dimension. Scale 1 stands for disagree and scale 7 for agree.

\begin{tabular}{|c|c|c|}
\hline Question & Item mean & Dim. mean \\
\hline $\begin{array}{l}\text { A - Enjoyment } \\
\text { (1) The interactive tag game made me laugh (more) than the normal tag game } \\
\text { (2) I would recommend the interactive tag game over the normal tag game } \\
\text { (3) I liked playing the interactive tag game more than the normal tag game } \\
\text { (4) I am more interested in further exploring the interactive tag games environment than playing normal tag } \\
\text { (5) I am more interested in playing the interactive tag game again than normal tag }\end{array}$ & $\begin{array}{l}5.22 \\
5.22 \\
5.33 \\
5.56 \\
5.52\end{array}$ & 5.37 \\
\hline $\begin{array}{l}\text { B - Immersion } \\
\text { (6) I felt more involved in the game when playing the interactive tag than when playing normal tag } \\
\text { (7) I was more engaged in the game when playing interactive tag than when playing normal tag } \\
\text { (8) I felt I lost track of time more when playing interactive tag than when playing normal tag } \\
\text { (9) I felt I was inside the game while playing interactive tag more than during normal tag }\end{array}$ & $\begin{array}{l}4.85 \\
4.89 \\
5.27 \\
4.70\end{array}$ & 4.93 \\
\hline $\begin{array}{l}\text { C - Gameplay } \\
\text { (10) I was able to anticipate what would happen next in response to the actions I initiated } \\
\text { (11) The controls for the game were appropriate } \\
\text { (12) The controls for the game felt natural } \\
\text { (13) I was able to clearly identify what game pieces/objects/models represented } \\
\text { (14) I experienced delay between my actions and the expected outcomes within the game } \\
\text { (15) I understood the graphics of the game }\end{array}$ & $\begin{array}{l}4.69 \\
5.81 \\
5.70 \\
5.81 \\
1.81(r) \\
5.93\end{array}$ & 4.96 \\
\hline $\begin{array}{l}\text { D - Enjoyment of game elements } \\
\text { (16) I enjoyed the graphics of the game } \\
\text { (17) I enjoyed the sound effects in the game } \\
\text { (18) I enjoyed the context and theme of the game }\end{array}$ & $\begin{array}{l}5.70 \\
4.69 \\
5.52\end{array}$ & 5.31 \\
\hline $\begin{array}{l}\text { Balance/fairness } \\
\text { (19) The game allowed me to demonstrate my ability of playing tag } \\
\text { (20) I think the game helps less skilled players } \\
\text { (21) I think the game aids skilled players } \\
\text { (22) The game interferes with my ability to play tag }\end{array}$ & $\begin{array}{l}4.11 \\
4.63 \\
4.04 \\
4.41(r)\end{array}$ & 4.30 \\
\hline $\begin{array}{l}\text { Skill level } \\
\text { (23) I am physically active } \\
\text { (24) I consider myself to be in good shape } \\
\text { (25) I exercise regularly } \\
\text { (26) I enjoy physical activity } \\
\text { (27) I consider myself a good tag player }\end{array}$ & $\begin{array}{l}4.70 \\
4.59 \\
4.26 \\
5.78 \\
4.81\end{array}$ & 4.83 \\
\hline
\end{tabular}

results show the dynamic circle size intervention is a valid strategy to balance skill levels without feeling unfair to the players.

5.1.1.6. Skill level. The Skill level category evaluates different aspects related to the physical capabilities of the players and their perceived ability to play tag. It is composed by the last five questions of the questionnaire (Q23-27). The mean for this category is $4.83(\sigma=1,12)$ and its Cronbach's alpha is 0.78 . The high alpha value suggests that this category could in fact be considered a dimension as the questions measure a single construct. This category explores how the perceived tag ability of the players correlates with the Enjoyment and Immersion dimensions. In this regard, we could not find any significant correlation between questions related to a player's physical abilities (Q23-26) and the Enjoyment or Immersion dimensions when using a 2-tailed bi-variate Pearson correlation test. Taking into account that the fitness level of the players varied greatly, we can say that the game is enjoyable independent of the physical conditioning of the players. We also did not find any correlation between Q27 and both dimensions, which shows that players' own rating of their skill is unrelated to whether they enjoyed the game.

\subsubsection{Observations and feedback}

Besides the questionnaire, we also invited players to a short discussion session. This discussion included elaborating on their impressions of the game and setup. We discuss the most important points that players brought up in the discussion, as well as our own remarks derived from observations of the game sessions. This information complements the evaluation using the questionnaires.
Given that one of the goals of the ITP was to allow players to exhibit natural play behavior, it was nice to witness an abundance of social interactions between the players. Players were yelling at each other, making jokes, making fun off, and seeking revenge when tagged. We also saw players exerting themselves significantly during the interactive tag game sub-sessions. Again, this signals that the interactive elements in the ITP allow players to physically exert themselves as they would in normal tag games.

When talking to the players about the interactive tag, it was interesting to see that while every player noticed the circles changing size during the game, most did not guess the reason behind the changes. Many players thought a player's speed was the reason for the size change. Nonetheless, speed was not considered at all for the intervention. This highlights two important points. First, we were able to change the game dynamics without affecting how players perceive the game. Second, the ITP made it possible to get the players position and role in real-time, allowing us to use this information to change game certain mechanics. This, in turn, shows how the ITP can facilitate the process of analyzing player behavior.

Although all players stated the interactive tag game was enjoyable and exhausting, there were some problems with the installation. The main problem was the network lag during the game sessions, which resulted in the circle moving slightly behind the players. Some players remarked that it was hard to control at the beginning, but as they got used to it, it added a strategic layer to the game as they had to predict where people were going. Another problem we encountered was that the sound effects of the game were not always noticed. The main purpose of the sounds was to notify the players whenever a tag took place, hopefully avoiding their need to look at the floor to check their circles. 


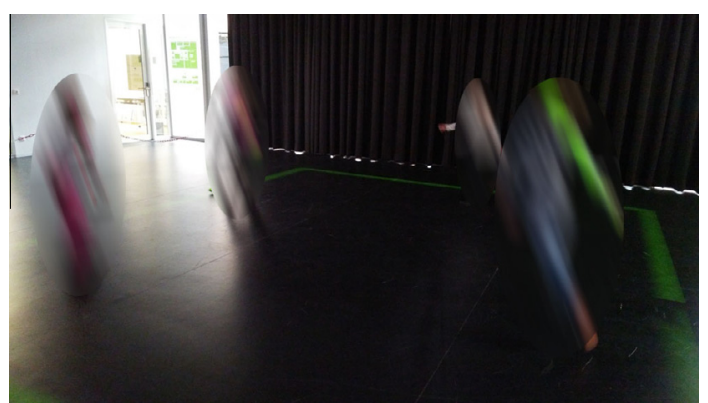

Fig. 7. Children playing traditional tag in the ITP.

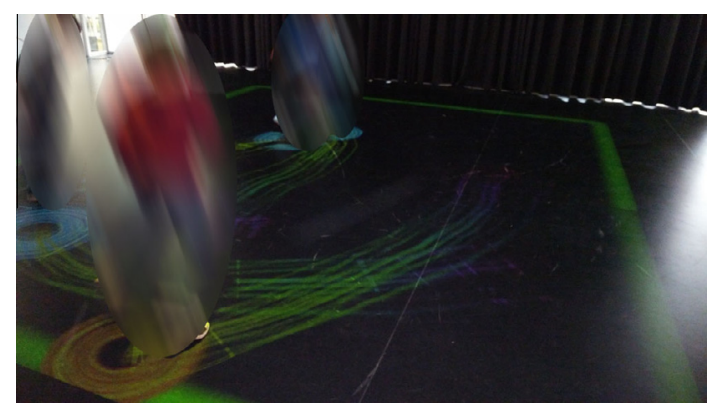

Fig. 8. Children playing interactive tag in the ITP.

\subsection{Second user study: children}

For our second user study, we invited children from two elementary schools to visit our university and take part in different activities, including playing tag in the ITP. The age of the children ranged between nine to twelve years. The user study was conducted over two consecutive mornings. Each school brought children from two different classes. We recorded a total of nineteen play sessions, each consisting of a traditional and an interactive tag sub-session (Figs. 7 and 8 respectively). We did not use any intervention for this study, and the size of the circles did not change. Also, the system lag from the first user study was corrected, and the audio effects were made more noticeable. Each sub-session lasted $90 \mathrm{~s}$. In all sessions, four players were randomly selected from the pool of available children to play together. The study has been approved by the university's ethical committee.

Information letters and consent forms were handed out to the parents by their teachers before the school visit. Every children in the school visit took part in the activities, but parents could give additional consent to let children participate in experiments and thus allow us to gather and use their data. Parents could also allow us to take photos or video recordings of the children for analysis purposes. Depending on the permissions granted by their parents, each child was given a label of a different color so that the researchers could identify them. As such, some children could play in the ITP and get their position and role data logged, but no photographs could be taken. Other children were only allowed to play, but no data could be recorded. In this last case, their play data was discarded. All children could only play once.

Once the color labels were arranged, children took part in any activity they liked. To recruit our participants, we went to the different stations and asked for children who wanted to participate in the game of tag. Once we found four, we led them to the ITP. There they were explained that they were going to play a game of tag (interactive or traditional, depending on the group's number). We alternated the initial condition between groups. Before each condition, we explained the game. For the interactive tag, this included two researchers playing interactive tag very briefly. The explanation served two purposes: inform the game's rules to the children, and give them time to rest between sub-sessions. The game started by asking the children to stand on one of the four corners of the playground, and then a countdown timer was displayed on the floor. We counted in real-time the number of tags per session to use in the evaluation of the ITP. After each sub-session, the children took part in a short, semi-structured interview. The interview that took place after the second sub-session was slightly longer as the players were also asked to give feedback and share their thoughts.

\subsubsection{Semi-structured interview}

We did not use a questionnaire to evaluate the play experience because our users were young children. Instead, we opted for a short, semi-structured interview. After each session, we asked the children whether they would like to play the game again. The answers they could give were yes, no or maybe. This was a quick and clear method to evaluate the enjoyment of the game, derived from the "Again-Again" table that has been used to evaluate perceived fun reasonably well for this target group [38]. After both sub-sessions, children were also asked what they liked or disliked about the ITP.

In general, children were very positive towards the interactive tag game. Out of 76 children, 75 indicated that they would have liked to play interactive tag again. Their responses were also immediate, with a lot of them eager to play again. Some children even begged to be allowed to play once more. On the other hand, a lot of the children were hesitant when asked whether they would like to play traditional tag again, with only 38 saying yes, 22 maybe, and 16 no. There was only one boy that indicated he liked the traditional tag game more than the interactive one. A lot of children indicated interesting reasons during the interviews about why they thought interactive tag was better than traditional tag without us mentioning those game aspects. We categorized the most frequent answers. The results are summarized in Table 3.

Four groups mentioned that novelty, in some way, played a role in them liking the ITP better. Six groups mentioned that interactive tag was more fun because it made it clear for everyone who the tagger was, which prevented them from wasting time discussing this. Another reason that was mentioned six times, especially by girls in mixed groups, was that the interactive tag game did not require physical contact, which prevented rough playing styles. Children from three groups said that simply the fact of it having digital gaming elements made it more fun. Three groups said that they preferred the interactive tag game because it was more challenging. Other reasons that were mentioned but not categorized were liking the circles/balls, or that you could get lucky as a result of tracking mistakes.

\subsubsection{Game observations}

The observations made during the traditional tag sub-sessions are similar to those we made during the recording of the Play corpus. Children were physically and socially invested in the game. Play broke down several times due to runners pretending they had not been tagged, taggers pretending they were runners to get close to other players and tag them, or due to discussions about who the tagger was. To try to solve this, children often verbally indicated that they tagged someone by saying "Tikkie!". Also, children sometimes reminded the group of whom was the current tagger. In one session, the children decided during the game to forbid tagging back the same player to make the game more fun.

In the interactive version of the game, we also saw intensive physical investment from the players, as well as social interactions between them. The amount of physical activity was comparable to what we observed in our ethnographic studies at schools. Many of 
Table 3

Results of the user study with children. On the left, the observed number of tags per session (Int stands for interactive tag, Trad for traditional tag). On the right, the results from the children's interview. The bottom row shows total and average values. The Fun column indicates the reasons for the ITP being more fun, as stated by the children ( $\mathrm{d}$ = digital, $\mathrm{h}=$ harder/more challenging, $\mathrm{nt}=$ no touch needed, $\mathrm{n}=$ novelty, $\mathrm{t}=$ tagger is always known, $-=$ nothing mentioned/non-categorized response).

\begin{tabular}{|c|c|c|c|c|c|}
\hline \multirow[t]{2}{*}{ Session } & \multicolumn{2}{|c|}{$\begin{array}{l}\text { Number of } \\
\text { tags }\end{array}$} & \multicolumn{2}{|c|}{$\begin{array}{l}\text { Again-again table }(\mathrm{Y}=\mathrm{Yes}, \mathrm{N}=\mathrm{No}, \\
\mathrm{M}=\text { Maybe })\end{array}$} & \multirow[t]{2}{*}{ Fun } \\
\hline & Int & Trad & Int & Trad & \\
\hline 1 & 4 & 17 & $4 \times Y$ & $3 \times \mathrm{M}, 1 \times \mathrm{Y}$ & - \\
\hline 2 & 10 & 11 & $4 \times Y$ & $4 \times \mathrm{M}$ & $\mathrm{d}, \mathrm{h}, \mathrm{nt}$ \\
\hline 3 & 8 & 17 & $4 \times Y$ & $4 \times \mathrm{M}$ & - \\
\hline 4 & 4 & 19 & $3 \times \mathrm{Y}, 1 \times \mathrm{M}$ & $3 \times \mathrm{M}, 1 \times \mathrm{Y}$ & $\mathrm{n}, \mathrm{h}$ \\
\hline 5 & 7 & 12 & $4 \times \mathrm{Y}$ & $2 \times \mathrm{N}, 2 \times \mathrm{Y}$ & $\mathrm{t}$ \\
\hline 6 & 5 & 17 & $4 \times Y$ & $4 \times \mathrm{N}$ & $\mathrm{t}, \mathrm{n}, \mathrm{d}$ \\
\hline 7 & 11 & 12 & $4 \times Y$ & $3 \times \mathrm{Y}, 1 \times \mathrm{N}$ & $t, n, d$ \\
\hline 8 & 4 & 16 & $4 \times Y$ & $3 \times \mathrm{Y}, 1 \times \mathrm{M}$ & nt \\
\hline 9 & 7 & 16 & $4 \times Y$ & $4 \times \mathrm{Y}$ & nt \\
\hline 10 & 6 & 17 & $4 \times Y$ & $3 \times \mathrm{Y}, 1 \times \mathrm{M}$ & $\mathrm{n}, \mathrm{nt}$ \\
\hline 11 & 8 & 19 & $4 \times Y$ & $4 \times \mathrm{M}$ & - \\
\hline 12 & 5 & 15 & $4 \times Y$ & $3 \times \mathrm{Y}, 1 \times \mathrm{M}$ & $\mathrm{h}$ \\
\hline 13 & 5 & 17 & $4 \times Y$ & $4 \times Y$ & $\mathrm{t}$ \\
\hline 14 & 6 & 16 & $4 \times Y$ & $4 \times N$ & nt \\
\hline 15 & 7 & 15 & $4 \times Y$ & $4 \times Y$ & - \\
\hline 16 & 6 & 21 & $4 \times Y$ & $3 \times \mathrm{N}, 1 \times \mathrm{Y}$ & nt \\
\hline 17 & 8 & 14 & $4 \times Y$ & $4 \times Y$ & $\mathrm{t}$ \\
\hline 18 & 4 & 21 & $4 \times Y$ & $3 \times \mathrm{Y}, 1 \times \mathrm{M}$ & $\mathrm{n}$ \\
\hline \multirow[t]{2}{*}{19} & 8 & 13 & $4 \times Y$ & $2 \times \mathrm{N}, 2 \times \mathrm{Y}$ & $\mathrm{t}$ \\
\hline & 6,5 & 16 & $75 \times \mathbf{Y}, \mathbf{1} \times \mathbf{M}$ & $38 \times Y, 22 \times M, 16 \times N$ & \\
\hline
\end{tabular}

Bold values represent the average/summary of the results.

the break-down of play scenarios were solved as the role was indicated clearly by their circle. Nonetheless, just like in the traditional version of the game, children kept reminding everyone who the tagger was from time to time. The ITP also introduces a tag-back cooldown, which prevented players from tagging each other constantly.

In several sessions children shortly exited the playing area trying to avoid being tagged, or fell down while running, which caused the tracker to lose them temporarily. When this happened to the tagger, the game assumed they had left the game and assigned a random player as the new tagger. Although we reminded them to stay inside the playing area, on average we saw about 2.6 of these role switches per game.

\subsection{Player behavior analysis and comparison}

The ITP is designed to facilitate the analysis of player behavior. This is achieved by logging player data in-game. Below, we present

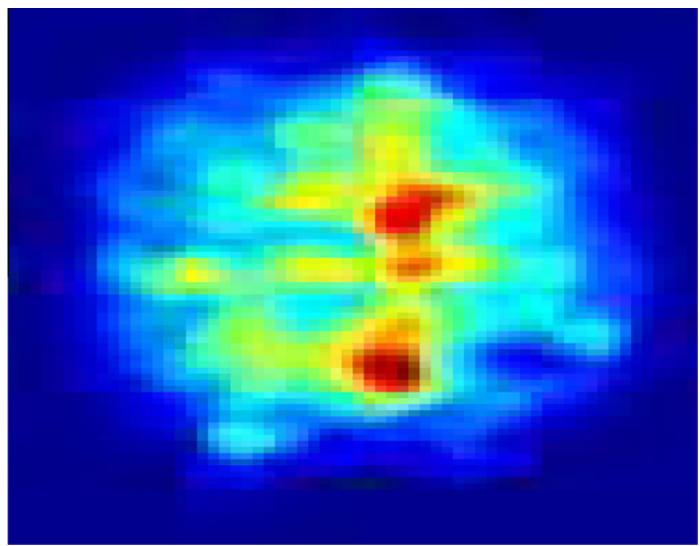

Fig. 9. Occupancy map of taggers when playing interactive tag for the adults.

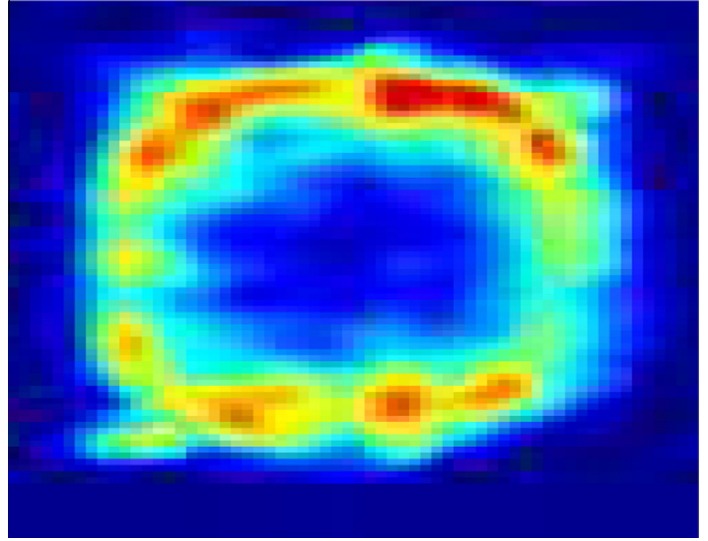

Fig. 10. Occupancy map of runners when playing interactive tag for the adults.

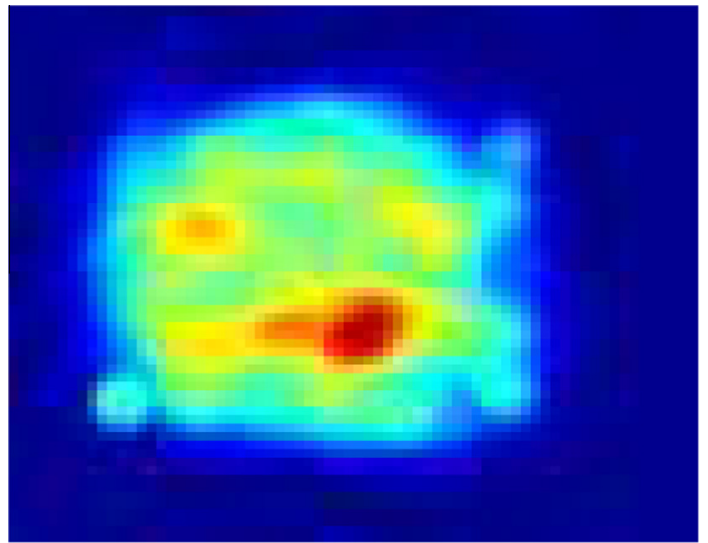

Fig. 11. Occupancy map of taggers when playing interactive tag for the children.

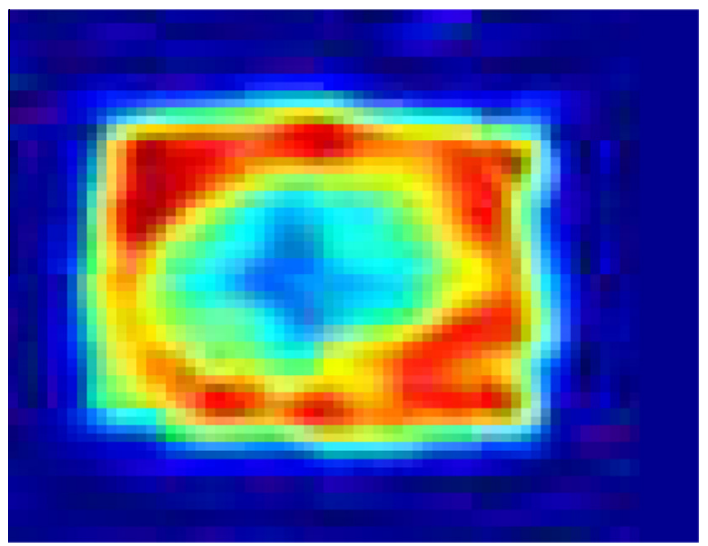

Fig. 12. Occupancy map of runners when playing interactive tag for the children.

the analysis of three behavioral cues derived from the players' position and role for adults and children.

\subsubsection{Player position}

The first cue indicates where players spent most of their time based on their role. As it can be seen in Figs. 9-12, taggers tend to stay around the center of the playground, minimizing their distance to the other players. Runners, on the other hand, stay near the border of the playground, maximizing their distance to the tagger and their chances of not being tagged. The behavior is largely the same for both age groups. 


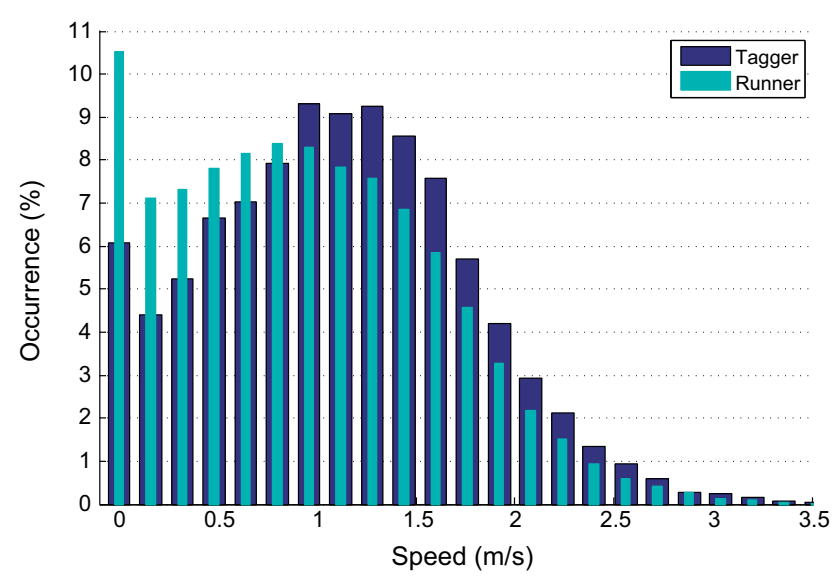

Fig. 13. Frequency of speeds at which adults moved during the interactive tag sessions.

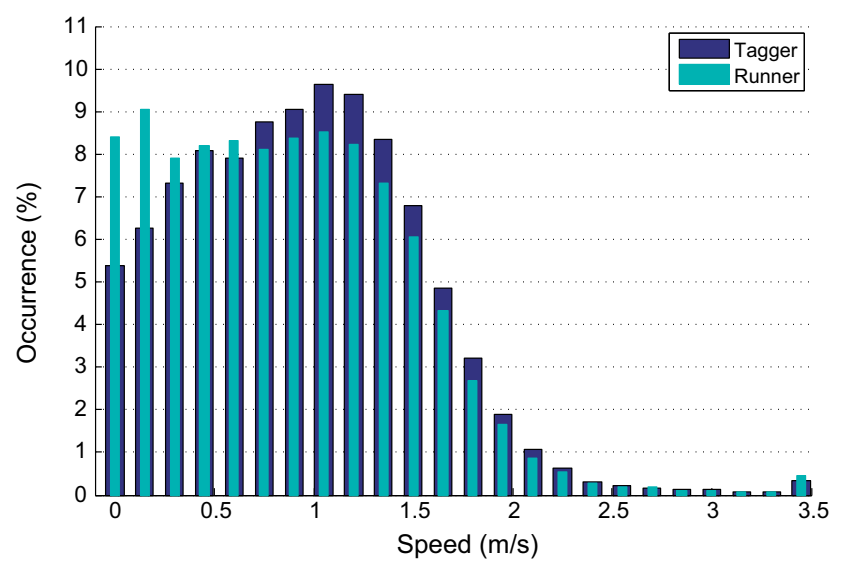

Fig. 14. Frequency of speeds at which children moved during the interactive tag sessions.

\subsubsection{Player speed}

The second cue is the speed at which the players move during the game. The frequency of player speeds, per role, are summarized in Figs. 13 and 14. The speed profiles for both user groups are very similar. The biggest differences can be found at speeds close to $0 \mathrm{~m} / \mathrm{s}$ and $1-2 \mathrm{~m} / \mathrm{s}$. Runners move more than taggers close to $0 \mathrm{~m} / \mathrm{s}$, which is probably due to runners being able to go to a corner, rest and wait until the tagger focuses them. On the other hand, taggers move at higher speeds more often than runners. In the case of the adults, runners moved at $1.01 \mathrm{~m} / \mathrm{s}$ and taggers at $1.18 \mathrm{~m} / \mathrm{s}$, on average. In the case of the children, runners moved

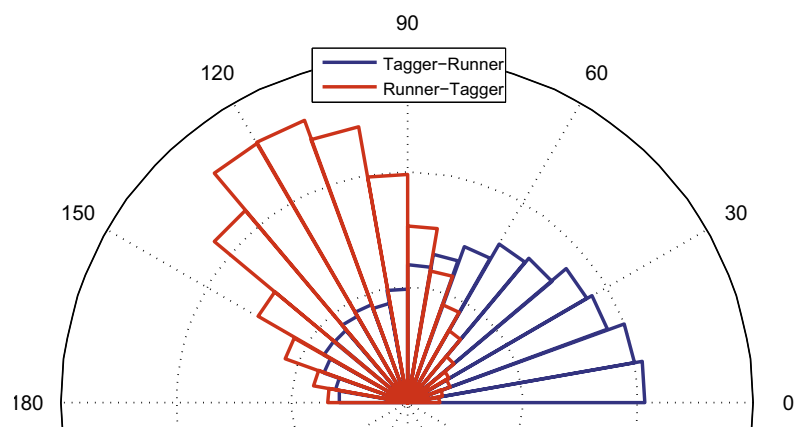

Fig. 15. Angular histogram of the relative movement direction between roles in the interactive tag sessions for the adults.

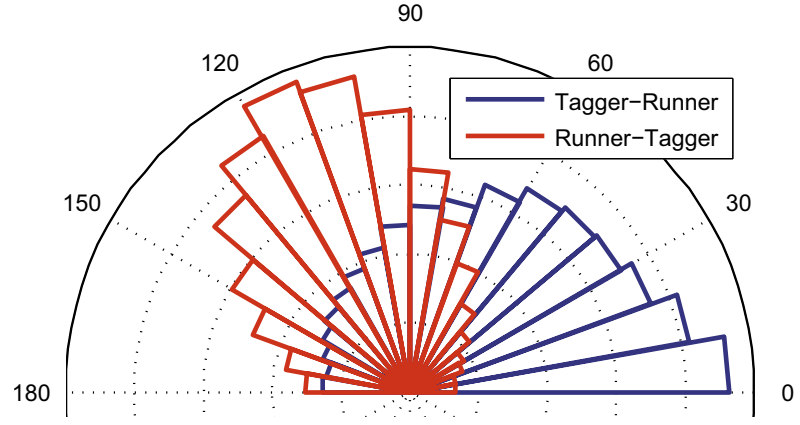

Fig. 16. Angular histogram of the relative movement direction between roles in the interactive tag sessions for the children.

at $0.89 \mathrm{~m} / \mathrm{s}$, taggers at $0.96 \mathrm{~m} / \mathrm{s}$. In general, taggers run slightly faster than runners in both age groups.

\subsubsection{Relative movement direction}

The third and last cue is the relative movement direction of players based on their roles. We look at the direction in which a player moves in relation to another player in the playground. Due to the rules of the game, taggers run towards runners, and runners run away from taggers. The relative movement direction angular histograms of players based on their roles can be seen in Figs. 15 and 16.

As we expected, the relative movement direction histogram for taggers with respect to runners has the highest counts for bins close to $0^{\circ}$. This means that taggers were mostly running towards runners. Runners ran away from taggers but, not in the completely opposite direction $\left(180^{\circ}\right)$, but at around $90-150^{\circ}$. The behavior is almost identical for both age groups. Therefore, there are no big differences between children and adults in the three cues that we have analyzed.

\section{Conclusions and future work}

When using interactive technology to enhance games, there is the risk of negatively affecting key characteristics inherent to play, such as the amount of social interactions or physical activity. In this paper, we have enhanced traditional tag without limiting players from exhibiting physically active, social behavior. We designed the Interactive Tag Playground, an interactive installation built with three goals in mind: enhance the tag game experience, allow players to physically and socially express themselves during play, and facilitate the analysis of player behavior. To evaluate whether these goals were met, we conducted two user studies with two age groups: adults and children. We also analyzed player behavior in both user studies using three behavioral cues to showcase the potential of the ITP as a research tool that facilitates behavior analysis.

Our studies showed that, overall, users preferred the interactive version of tag over the traditional one. In the first user study, the two dimensions of the questionnaire used to compare the game experience, Enjoyment and Immersion, showed a statistically significant preference towards the interactive tag game. In our second user study, virtually all children wanted to play again interactive tag, whereas half of them wanted to play traditional tag again. Some of the players stated that novelty played a role in their decision, but other reasons were mentioned as well, such as the presence of game state information, or the game being more challenging. In both studies we witnessed an abundance of social interactions, as well as high levels of physical activity. When we 
analyzed the behavior exhibited by adults and children, there were almost no differences between them. This means the ITP manages to enhance the game experience, support the physical and social aspects of play, and facilitate the behavior analysis process.

With these three goals met, we believe much more can be accomplished using the technology at hand. For instance, in our first user study, we showed that the ITP's real-time player data collection can be used to implement skill balancing strategies without players feeling cheated by the game. Besides skill balancing, we would like to explore interventions to restore engagement, steer behavior, modulate physical activity, or promote social interactions between players. Another interesting research direction is the evaluation of physical activity or social interactions using objective and automatically collected information from the ITP. Since the level of physical activity that we observed during interactive tag game sessions was similar to what we have observed in tag game ethnographic studies, it would be interesting to use the ITP to objectively evaluate to what extent this is true. Based on our results, we feel confident that the ITP is an entertainment platform capable of enhancing games while facilitating opportunities for in-game, automatic analysis of player behavior or post hoc evaluation of goals.

\section{Acknowledgment}

This publication was supported by the Dutch national program COMMIT.

\section{References}

[1] L.A. Barnett, Developmental benefits of play for children, J. Leisure Res. 22 (2) (1990) 138-153.

[2] J. Isenberg, N. Quisenberry, Play: essential for all children. A position paper of the association for childhood education international, Childhood Educ. 79 (1) (2002) 33-39.

[3] T.Z. Henderson, D.J. Atencio, Integration of play, learning, and experience: what museums afford young visitors, Early Childhood Educ. 35 (3) (2007) 245-251.

[4] N. Canning, Children's empowerment in play, Eur. Early Childhood Educ. Res. J. 15 (2) (2007) 227-236.

[5] M.J. Guralnick, M.A. Hammond, R.T. Connor, B. Neville, Stability, change, and correlates of the peer relationships of young children with mild developmental delays, Child Develop. 77 (2) (2006) 312-324.

[6] K.R. Ginsburg, The importance of play in promoting healthy child development and maintaining strong parent-child bonds, Pediatrics 119 (1) (2007) 182-191.

[7] A.D. Pellegrini, The Role of Play in Human Development, Oxford University Press, 2009.

[8] E.A. Vandewater, H.M. Cummings, The Handbook of Children, Media, and Development, Ch. Media Use and Childhood Obesity, Blackwell Publishing Ltd., Oxford, UK, 2008. pp. 355-380.

[9] S. Turkle, Alone Together: Why We Expect More from Technology and Less from Each Other, Basic Books, 2011.

[10] E.A. Vandewater, M. Shim, A.G. Caplovitz, Linking obesity and activity level with children's television and video game use, J. Adolescence 27 (1) (2004) 71-85.

[11] W. Peng, J.C. Crouse, J.-H. Lin, Using active video games for physical activity promotion: a systematic review of the current state of research, Health Educ. Behav. 40 (2) (2013) 171-192.

[12] K. Isbister, How to stop being a buzzkill: designing yamove!, a mobile tech mash-up to truly augment social play, in: Proceedings of International Conference on Human-Computer Interaction with Mobile Devices and Services, 2012 , pp. $1-4$

[13] A. Moreno, R. van Delden, R. Poppe, D. Reidsma, Socially aware interactive playgrounds, IEEE Pervasive Comput. 12 (3) (2013) 40-47.

[14] R. van Delden, A. Moreno, D. Reidsma, R. Poppe, D. Heylen, Steering gameplay behavior in the interactive tag playground, in: Proceedings of European Conference on Ambient Intelligence, 2014, pp. 145-157.
[15] T. Bekker, E. Hopma, J. Sturm, Creating opportunities for play: the influence of multimodal feedback on open-ended play, Int. J. Arts Technol. 3 (4) (2010) 325-340.

[16] R. Poppe, R. van Delden, A. Moreno, D. Reidsma, Interactive playgrounds for children, in: A. Nijholt (Ed.), Playful User Interfaces, Springer Verlag, 2014, pp. 99-118.

[17] N. Bianchi-Berthouze, Understanding the role of body movement in player engagement, Human-Comput. Interact. 28 (1) (2013) 40-75.

[18] B.A.M. Schouten, R. Tieben, A. van de Ven, D.W. Schouten, Computer Analysis of Human Behavior, Ch. Human Behavior Analysis in Ambient Gaming and Playful Interaction, Springer, 2011. pp. 387-403.

[19] F. Mueller, K. Isbister, Movement-based game guidelines, in: Proceedings of the Conference on Human Factors in Computing Systems, 2014, pp. 2191-2200.

[20] F. Mueller, D. Altimira, R.A. Khot, Reflections on the design of exertion games, Games Health J. 4 (1) (2015) 3-7.

[21] D. Tetteroo, D. Reidsma, E.M.A.G. van Dijk, A. Nijholt, Yellow is mine!: designing interactive playgrounds based on traditional children's play, in: A.D. Cheok, A. Nijholt, T. Rom?o (Eds.), Entertaining the Whole World, Springer Verlag, 2014, pp. 63-84.

[22] A. Moreno, R. Poppe, Automatic behavior analysis in tag games: from traditional spaces to interactive playgrounds, J. Multimodal User Interf. 10 (1) (2016) 63-75.

[23] A. Moreno, R. van Delden, R. Poppe, D. Reidsma, D. Heylen, Augmenting traditional playground games to enhance game experience, in: Proceedings of the International Conference on Intelligent Technologies for Interactive Entertainment, 2015, pp. 140-149.

[24] T. Avontuur, R. de Jong, E. Brink, Y. Florack, I. Soute, P. Markopoulos, Play it our way: customization of game rules in children's interactive outdoor games, in: Proceedings of the Interaction Design and Children Conference, 2014, pp. 95-104.

[25] F. Mueller, M. Gibbs, F. Vetere, An exploration of exertion in mixed reality systems via the "Table Tennis for Three" game, in: E. Dubois, P. Gray, L. Nigay (Eds.), The Engineering of Mixed Reality Systems, Springer, London, 2009, pp. 165-182.

[26] C. Stockhausen, J. Smyzek, D. Krömker, Beats down: using heart rate for game interaction in mobile settings, in: Proceedings of the International Conference on Human-Computer Interaction - INTERACT, 2013, pp. 523-530.

[27] P. Navarro, M.L. Johns, T.-H. Lu, H. Martin, V. Poduval, M. Robinson, A. Roxby, M.G. Christel, Webz of war: a cooperative exergame driven by the heart, in: Proceedings of the International Games Innovation Conference, 2013, pp. 187-190.

[28] P. Landry, N. Parés, Controlling and modulating physical activity through interaction tempo in exergames: a quantitative empirical analysis, J. Ambient Intell. Smart Environ. 6 (3) (2014) 277-294.

[29] D. Altimira, M. Billinghurst, F. Mueller, Understanding handicapping for balancing exertion games, in: Extended Abstracts of the Conference on Human Factors in Computing Systems, 2013, pp. 1125-1130.

[30] T. Stach, T.C.N. Graham, J. Yim, R.E. Rhodes, Heart rate control of exercise video games, in: Proceedings of Graphics Interface, 2009, pp. 125-132.

[31] R. Vicencio-Moreira, R.L. Mandryk, C. Gutwin, Balancing multiplayer firstperson shooter games using aiming assistance, in: Proceedings of the Games, Entertainment and Media Conference, 2014, pp. 1-8.

[32] J. Sturm, T. Bekker, V. Vanden Abeele, S. Johansen, M. van Kuijk, B. Schouten, Playful interactions stimulating physical activity in public spaces, in: Proceedings of the Conference on Human Factors in Computing Systems Workshops, 2013, pp. 1-6.

[33] S.E. Lindley, J. Le Couteur, N.L. Berthouze, Stirring up experience through movement in game play: effects on engagement and social behaviour, in: Proceedings of the Conference on Human Factors in Computing Systems, 2008, pp. 511-514.

[34] M. Pasch, N. Bianchi-Berthouze, B. van Dijk, A. Nijholt, Movement-based sports video games: investigating motivation and gaming experience, Entertain. Comput. 1 (2) (2009) 49-61.

[35] T. Bekker, L. de Valk, B. Eggen, A toolkit for designing playful interactions: the four lenses of play, J. Ambient Intell. Smart Environ. 6 (3) (2014) 263-276.

[36] L. op't Hof, J. de Pee, J. Sturm, T. Bekker, J. Verbeek, Prolonged play with the colorflares: how does open-ended play behavior change over time?, in: Proceedings of the International Conference on Fun and Games, 2010, pp 99106.

[37] R.E. Kalman, A new approach to linear filtering and prediction problems, J. Basic Eng. 82 (1) (1960) 35-45.

[38] J. Read, S. MacFarlane, Using the fun toolkit and other survey methods to gather opinions in child computer interaction, in: Proceedings of the Conference on Interaction Design and Children, 2006, pp. 81-88. 